\title{
Support of acute lymphoblastic leukemia cells by nonmalignant bone marrow stromal cells
}

\author{
SANA USMANI, URMILA SIVAGNANALINGAM, OLENA TKACHENKO, LETI NUNEZ, \\ JESSICA C. SHAND and CRAIG A. MULLEN
}

Division of Pediatric Hematology/Oncology, Department of Pediatrics, University of Rochester, Rochester, NY 14620, USA

Received July 17, 2018; Accepted February 22, 2019

DOI: $10.3892 / \mathrm{ol} .2019 .10188$

\begin{abstract}
The present report describes work examining the manner in which nonmalignant bone marrow stromal cells prevent acute lymphoblastic leukemia (ALL) cell death. The initial focus was on the role of stromal cell-derived C-X-C motif chemokine 12 (CXCL12). Interference with CXCL12 production by stroma or blockade of its interactions with ALL by plerixafor did increase ALL cell death and in sensitive ALLs there was synergistic effect with conventional chemotherapy drugs. However, in contrast to most reports, there was considerable heterogeneity regarding the effect between 7 unique primary ALLs, with several exhibiting no sensitivity to CXCL12 blockade. The diversity in effect was not explained by differences in the expression of ALL cell surface receptors for CXCL12. The modest and variable effects of interference with CXCL12 on ALL led to the assessment of gene expression profiles of stromal cells and ALL cells. Gene set enrichment analysis identified pathways associated with metabolism and redox reactions as potentially important in the stromal cell: leukemia cell interaction. Exploratory imaging studies demonstrated bidirectional transfer of intracellular calcien-labelled molecules and also bidirectional transfer of mitochondria between stromal cells and ALL cells, providing potential means of metabolic interdependence of stromal cells and ALL cells.
\end{abstract}

\section{Introduction}

Cancer cells have complex interrelationships with nonmalignant cells in their tissue microenvironments. In a variety

Correspondence to: Dr Craig A. Mullen, Division of Pediatric Hematology/Oncology, Department of Pediatrics, University of Rochester, Box 777, 601 Elmwood Avenue, Rochester, NY 14620, USA

E-mail: craig_mullen@urmc.rochester.edu

Abbreviations: ALL, acute lymphoblastic leukemia; GFP, green fluorescent protein

Key words: acute lymphoblastic leukemia, CXCL12, plerixafor, chemotherapy, stromal cells, bone marrow microenvironment of cancers, non-malignant cells have been shown to exhibit complex effects on malignant cells with activities that both promote and inhibit cancer cell growth. In addition, the tumor microenvironment has been shown to influence the effect of anticancer drugs on cancer cells, and in many circumstances has been shown to exert some protective effect on cancer cells. Leukemia is a malignancy of bone marrow origin. The bone marrow microenvironment is a complex, highly vascular tissue. Hematopoietic cells in the marrow are derived from hematopoietic stem cells, while nonhematopoietic stromal cells are derived from mesenchymal stem cells $(1,2)$.

Acute lymphoblastic leukemia (ALL) is a cancer derived from lymphoid precursors in the bone marrow, most commonly B cell precursors. Although it is a high grade malignancy that grows rapidly in vivo, primary ALL cells do not grow well in vitro, and spontaneously undergo apoptosis. However, primary ALL cells do survive in vitro when cocultured with nonmalignant bone marrow stromal cells $(3,4)$. Bone marrow stromal cells are nonhematopoietic cells in the bone marrow that are derived from mesenchymal stem cells. Functionally they are defined by their adherence to plastic in standard tissue culture conditions. Phenotypically they are negative for hematopoietic cell markers CD45, CD34, CD14, CD11b, CD79, CD19 and HLA-DR (5). The mechanisms explaining the leukemia cells' stromal dependence are not well understood. Stromal cell-derived chemokines are among the mechanisms studied. Work by a number of groups has shown that the chemokine CXCL12 plays a role in hematopoietic precursor cell homing to and retention in bone marrow (6-9) and can affect ALL cells. There is interest in developing leukemia therapies that target CXCL12.

In the present study we examined the impact of interference of the CXCL12 effect on a panel of recently derived patient-derived xenograft ALLs. While we were able to reproduce results of others that interference with CXCL12 could affect ALL survival, we observed considerable variation in the effect within our panel of patient-derived xenografts. These results led us to more broadly examine gene expression patterns in stromal cells and leukemia cells. We discovered overexpression of pathways related to redox reactions and metabolism. We then discovered that the stromal cells and leukemia cells directly exchange intracellular materials and mitochondria. 


\section{Materials and methods}

ALL cells. Deidentified primary B lineage ALL cells from adult and pediatric patients were obtained from bone marrow or peripheral blood leukapheresis samples at time of initial diagnosis or relapse. The samples were used under the auspices of an IRB approved protocol. The IRB deemed that individual patient consent was not needed since no personal identifying information was involved and the materials were from residual lab samples that would otherwise have been discarded. All samples were from patients who met NCI criteria for high risk ALL. Limited clinical, genetic and phenotypic data are available because of the deidentification process. All specimens were human CD45 positive, human CD19 positive both before and after expansion in immunodeficient mice. Table I contains information about individual samples.

Specimens were expanded a single time as patient-derived xenografts in NOD-SCID mice. Only first-generation xenograft samples were used; i.e., leukemias were not serially passaged in mice. At 8-12 weeks of age, mice received 250 cGy total body irradiation. Mice were injected intravenously with $5 \times 10^{6}$ leukemia cells $4 \mathrm{~h}$ later. The spleen and bone marrow were harvested after 8-12 weeks. To confirm engraftment of leukemic cells, the cells were examined by flow cytometry with human CD19 and CD45 antibodies. We compared ALL cells before and after xenograft expansion and found no differences in flow phenotype, morphology, stromal dependence and in vitro growth potential.

Established cell lines. SC is a human monocyte/macrophage cell line obtained from ATCC (ATCC CRL-9855). Subsequently, STR profiling (Genetica LabCorp, Burlington, NC, USA) and STR analysis was performed using the ATCC STR database (https://www.atcc.org/en/STR_Database.aspx), which demonstrated that the cell line was derived from U-937, a human histiocytic lymphoma (ATCC CRL-1593.2). Jurkat is a human T lymphoblastic leukemia line. K562 is a human chronic myelogenous leukemia line. Sup T1 is a human T lymphoblastic lymphoma line.

Stromal cells. In order to have consistent and uniform marrow stromal cell source we used a stromal cell line-derived from normal bone marrow immortalized with the human telomerase reverse transcriptase (TERT) gene (10). Unless otherwise specified in the text this is the stromal line used in an experiment. A second immortalized stromal line, HS27 (ATCC CRL-2496), derived from normal bone marrow and immortalized with a retroviral vector containing human papilloma virus E6/E7 genes was also used (11). Short term marrow stromal cultures were frequently used to confirm observations made with the immortalized stromal lines. These primary stromal cell cultures were established by placing 1-3 $\mathrm{ml}$ of marrow aspirate in $10 \mathrm{ml}$ RPMI supplemented with $20 \%$ FCS, MEM non-essential amino acids $1 \mathrm{X}$, sodium pyruvate $1 \mathrm{mM}$, 2-mercaptopurine $5.5 \mu \mathrm{M}$, penicillin/streptomycin $1 \mathrm{X}$ and $1 \mu \mathrm{M}$ hydrocortisone $(\mathrm{R} 10 \mathrm{C}+\mathrm{H})$ in $25 \mathrm{~cm}^{2}$ conventional tissue culture flasks. After 24-48 h nonadherent cells were removed. At first passage (14-21 days) FCS was reduced to $10 \%$. Stromal cells were also generated in a similar manner from
Table I. Characteristics of ALL cells used in these studies.

\begin{tabular}{lcccc}
\hline Name & Tissue & Age & $\begin{array}{c}\text { Disease } \\
\text { phase }\end{array}$ & Cytogenetics \\
\hline ALL A & Blood & Adult & Diagnosis & Unknown \\
ALL B & Blood & Adult & Diagnosis & Unknown \\
ALL C & Blood & Pediatric & Diagnosis & Ph + \\
ALL D & Marrow & Pediatric & Relapse & MLL-AF4 \\
ALL E & Blood & Adult & Relapse & Unknown \\
ALL F & Blood & Adult & Diagnosis & Unknown \\
ALL G & Marrow & Pediatric & Relapse & Normal \\
\hline
\end{tabular}

ALL, acute lymphoblastic leukemia; $\mathrm{Ph}+$, Philadelphia chromosome $\mathrm{t}(9 ; 22)(\mathrm{q} 34 ; \mathrm{q} 11)$; MLL-AF4, $\mathrm{t}(4 ; 11)(\mathrm{q} 21 ; \mathrm{q} 23)$ involving the MLL and $\mathrm{AF} 4$ genes.

de-identified human skin, fat, and placenta obtained under an IRB approved protocol from discarded surgical material. Freshly isolated tissues were minced to $2 \mathrm{~mm}$ fragments, placed in culture and handled as above. Primary stromal cells were used within 4 passages. Stromal cells did not express hematopoietic genes PTPRD (CD45), CD34, CD19 or CD79a. All were adherent and expressed COL1A2 (collagen1). The stromal cells used were not mesenchymal stem cells since they did not meet consensus criteria (5) for multipotent mesenchymal stem cells (i.e., ENG positive, THY-1 positive, NT5E positive).

Counting live ALL cells by flow cytometry. A total of $5 \times 10^{3}$ stromal cells were plated in flat-bottom 96-well plates in $\mathrm{R} 10 \mathrm{C}+\mathrm{H}$. Two days later medium was removed and viable ALL cells were added in AIM V medium (Thermo Fisher Scientific, Inc., Waltham, MA, USA). A total of $3 \times 10^{4}$ leukemia cells were added per well in experiments. Five days later wells were harvested and flow cytometry was performed by 3-color flow cytometry (huCD19-FITC+, huCD45-PE dim+, AAD-, plus $2.5 \times 10^{5}$ counting beads). To create consistency within each experiment, sample acquisition continued until a fixed number of bead events were collected (this number varied from experiment to experiment but ranged between $2 \times 10^{4}$ and $3 \times 10^{4}$ ). For analysis gating was made on the huCD19+huCD45+ region and the number of AAD-live cells reported.

Vital dye transfer studies. Cells were incubated at $37^{\circ} \mathrm{C}$ for 30 min with calcein AM $1 \mu \mathrm{M}$ (BD Pharmingen; BD Biosciences, Franklin Lakes, NJ, USA) as directed by manufacturer. Following extensive washing they were placed in culture. One day later the cells were collected and calcein labeled cells were identified by flow cytometry. To stain mitochondria, cells were incubated per manufacturer's instructions in MitoTracker Red CMXRos $25 \mathrm{nM}$ (Invitrogen; Thermo Fisher Scientific, Inc.). Labeled cells were imaged by fluorescent microscopy. In experiments assessing the need for cell:cell contact, stromal cells were plated on the surface of 12-well tissue culture plates. $0.4 \mu \mathrm{m}$ membrane cell culture Transwell inserts (Falcon) were then 
placed in wells, and leukemia cells placed in the Transwell insert.

Stromal cell treatment with siRNA. Stromal cells in 96-flat bottom well plates were reverse transfected with 6 pmol Stealth siRNA (Invitrogen; Thermo Fisher Scientific, Inc.) specific for the target gene or control siRNA, using Lipofectamine RNAiMAX $0.05 \mu \mathrm{M}$. Wells were washed 2 days later with RPMI or AIM V and then used in ALL survival assays. Effectiveness of target gene knockdown was measured at $48 \mathrm{~h}$ after transfection by quantitative SYBR-Green RT-PCR.

Drug treatment of cultures. Drugs were added to cocultures at the time leukemia cells were added and remained present for the full 5 days of coculture. The concentrations used were: dexamethasone $6 \mathrm{ng} / \mathrm{ml}$, methotrexate $6.25 \mathrm{ng} / \mathrm{ml}$, vincristine $3 \mathrm{ng} / \mathrm{ml}$, 6-mercaptopurine $250 \mu \mathrm{M}$, plerixafor 200-400 $\mu \mathrm{M}$, recombinant human CXCL12 240 ng/ml. Drug interaction was analyzed using the fractional product method in which one compares the predicted magnitude of combined drug treatment with the actual results (12). For example, if drug $X$ reduced the leukemia cell population to 0.75 , and drug $\mathrm{Y}$ to 0.75 , the fractional product prediction for combined treatment would be $0.75 \times 0.75=0.56$. If the actual effect of combined treatment was 0.4 , drug synergy (as opposed to simple additive effects) might be present.

Gene expression analyses. RNA was prepared from $5 \times 10^{6}$ cells using Qiagen RNeasy kits (Qiagen, Inc., Valencia, CA, USA). For experiments in which assessment of gene expression changes after stroma cell:leukemia cell occurred, $1.6 \times 10^{6}$ stromal cells were plated in a $25 \mathrm{~cm}^{2}$ flask and $24 \mathrm{~h}$ later $5 \times 10^{6}$ leukemia cells were plated. Two days later the cells were harvested and purified by flow cytometric sorting. Residual genomic DNA was removed by DNAse treatment and a cDNA library was prepared. Sequencing was performed in the University of Rochester Genomics Core Facility (Rochester, NY, USA). Sequencing was performed using Illumina HiSeq 2500 Sequencer (Illumina Inc., San Diego, CA, USA). Raw reads were demultiplexed using configurebcl2fastq. $\mathrm{pl}$ version 1.8.4. Quality filtering and adapter removal were performed using Trimmomatic version 0.32 with the following parameters: 'SLIDINGWINDOW:4:20 TRAILING:13 LEADING:13 ILLUMINACLIP:adapters. fasta:2:30:10 MINLEN:15'. Processed/cleaned reads were then mapped to the human reference genome (hg19) using SHRiMP version 2.2.3 with the following parameters: '--qv-offset 33 --all-contigs'. Gene-level read quantification and differential expression analysis were performed using Cufflinks version 2.0.2 (cuffdiff) with these parameters: '--FDR 0.05 -u -b GENOME' (thus employing a correction for multiple comparisons). For further gene set enrichment analysis only those differential expressed genes with both $>0.1$ fragments per kilobase per million (FPKM) and a two-fold up or down regulation were considered. Functional annotation clustering of differentially expressed genes was performed using the web-based software of the Gene Ontology Consortium (geneontology.org. PANTHER Overrepresentation test release 20170413, GO Ontology database release 2017-06-29, Homo sapiens reference list, and use of a Bonferroni correction for identification of statistically significant overrepresentation.)

Statistics. Statistical analyses were performed using $\mathrm{R}$ statistical language and RStudio. Data from leukemia cell survival assays was found to be normally distributed. In cases in which there were more than two groups, one way analysis of variance was performed; the Tukey post hoc test was applied to determine whether there were significant differences between specific pairs of experimental groups. These P-values (which adjust for multiple comparisons) are reported in the text and figures. For studies of CXCL12 expression and leukemia cell survival data were not normally distributed. Percent survival leukemia on a supporting cell type was first normalized to leukemia survival on our internal standard stromal line, and then log transformed. CXCL12 gene expression was expressed as FPKM, and then divided by HLA-A FKPM (to provide internal control). This normalized expression was then log transformed. A Spearman correlation test was then performed.

\section{Results}

Relationship between CXCL12 gene expression and support of ALL cells. Work by a number of groups has shown that the chemokine CXCL12 plays a role in hematopoietic precursor cell homing to and retention in bone marrow (6-9). We assessed CXCL12 gene transcription in 17 stromal cell lines that supported ALL cell survival in coculture and in 7 cell lines derived from hematopoietic cells that poorly support leukemia cell survival in coculture (Table II has details about these cells). We then explored correlation of percent survival of ALL cells with CXCL12 expression (as normalized to HLA-A expression). The Spearman correlation (rho) was $0.542(\mathrm{P}=0.0092)$ indicating a moderate positive correlation between the two (data not shown).

Interference with CXCL12 produces a modest reduction in survival of leukemia cells. To assess a causal relationship between stromal cell CXCL12 expression and leukemia cell survival we transfected stromal cells with siRNA specific for CXCL12 or control siRNA. Quantitative RT-PCR studies showed CXCL12 knockdown efficiency was typically in the range of $50-75 \%$ (data not shown). Fig. 1 shows a modest (10-25\%) reduction of leukemia cell survival due to CXCL12 knockdown.

It is possible that these experiments underestimated the role of CXCL12 because siRNA knockdown was incomplete. CXCL12 interacts with the chemokine receptor CXCR4 on hematopoietic cells. Plerixafor is a small molecule inhibitor of this interaction $(13,14)$. Some studies of plerixafor have shown that it leads hematopoietic cells to mobilize away from stroma, and can increase the antileukemia activity of some drugs. Use of plerixafor would allow us to more fully interfere with CXCL12 effects on leukemia cells. We added plerixafor to the stromal cell-leukemia cell cocultures. We found that plerixafor reduced the survival of 5 of 7 independent ALLs (Fig. 2), but the effect ranged significantly between leukemias (20-80\%). Two of the ALLs showed no reduction in survival. In independent experiments we found that nonma- 
Table II. Characteristics of cells assessed for capacity to support ALL cells in vitro.

\begin{tabular}{|c|c|c|}
\hline $\begin{array}{l}\text { Supports } \\
\text { leukemia }\end{array}$ & Cell type & Cell name \\
\hline Yes & Marrow-derived primary stroma & $\mathrm{p} 210$ \\
\hline Yes & Marrow-derived primary stroma & p235 \\
\hline Yes & Marrow-derived primary stroma & p364 \\
\hline Yes & Marrow-derived primary stroma & p386 \\
\hline Yes & Marrow-derived primary stroma & p395 \\
\hline Yes & Marrow-derived primary stroma & p416 \\
\hline Yes & Marrow-derived primary stroma & p417 \\
\hline Yes & $\begin{array}{l}\text { Subclone of hTERT } \\
\text { immortalized stroma line }\end{array}$ & cl_19 \\
\hline Yes & $\begin{array}{l}\text { Subclone of hTERT } \\
\text { immortalized stroma line }\end{array}$ & cl_23 \\
\hline Yes & $\begin{array}{l}\text { Subclone of hTERT } \\
\text { immortalized stroma line }\end{array}$ & cl_30 \\
\hline Yes & $\begin{array}{l}\text { Subclone of hTERT } \\
\text { immortalized stroma line }\end{array}$ & cl_33 \\
\hline Yes & Adipose-derived primary stroma & Adipose \\
\hline Yes & Skin-derived fibroblastic stroma line & Fibroblast \\
\hline Yes & hTERT immortalized stroma line & P15 \\
\hline Yes & Placenta-derived primary stroma & Placenta \\
\hline Yes & Skin-derived primary stroma & Skin \\
\hline Yes & Skin-derived primary stroma & Skin \\
\hline No & B ALL & ALL C \\
\hline No & B ALL & ALL B \\
\hline No & B ALL & ALLA \\
\hline No & T acute lymphoblatsic leukemia line & Jurkat \\
\hline No & Chronic myelogenous leukemia line & K562 \\
\hline No & Monocyte/macrophage cell line & $\mathrm{SC}$ \\
\hline No & Histiocytic lymphoma & U-937 \\
\hline No & T lymphoblast lymphoma & SupT1 \\
\hline
\end{tabular}

ALL, acute lymphoblastic leukemia; hTERT, human telomoerase gene.

lignant hematopoietic bone marrow cells did not exhibit the same degree of dependence on stroma, and that plerixafor did not significantly reduce survival of these normal marrow cells (data not shown).

One possible explanation for the variability in the effect of plerixafor could be differences in expression of receptors for CXCL12 on leukemia cells. We performed flow cytometry for both CXCR4 and CXCR7 (receptors for CXCL12) $(15,16)$ on the ALLs. We found similar levels of both receptors on both plerixafor sensitive and insensitive leukemia cells (Fig. 3).

Effect of plerixafor on antileukemia activity of commonly used leukemia drugs. We then explored whether interference of stromal support with plerixafor would affect the antileukemia activity of commonly used chemotherapy drugs (mercaptopurine, methotrexate, dexamethasone and vincristine). Initially we performed dose-response
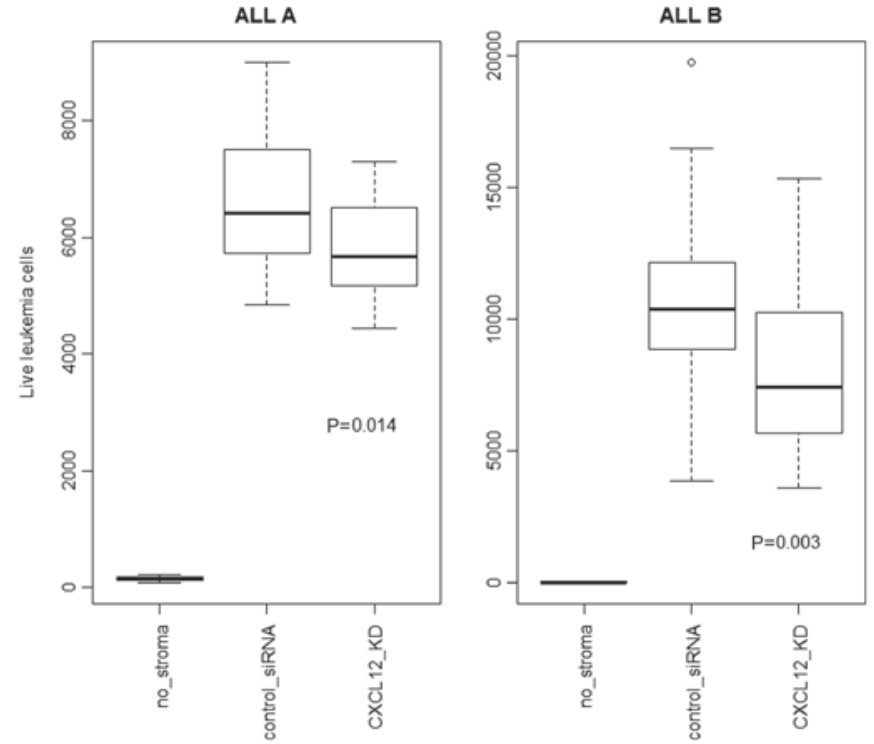

Figure 1. Knockdown of stromal CXCL12 expression leads to reduced ALL survival in coculture. Stromal cells were transfected with siRNA for CXCL12 or control siRNA. In each panel the left bar is no stroma $(n=12)$, the middle bar is stroma transfected with control siRNA $(n=18-24)$, and the left bar is the siRNA for CXCL12 $(n=20-23)$. Box and whisker plots represent the data. P-values are for comparisons of control siRNA to CXCL12 siRNA. CXCL12, C-X-C motif chemokine ligand 12; ALL, acute lymphoblastic leukemia; siRNA, small interfering RNA.

experiments with commonly used ALL chemotherapy drugs. We then performed coculture assays in which we combined plerixafor and conventional chemotherapy drugs. We used concentrations of conventional chemotherapy drugs that were likely to produce modest or minimal cytotoxicity alone because that would allow us to detect either additive or synergistic activity with plerixafor. We performed the assays with three unique ALLs, two of which had shown significant sensitivity to plerixafor alone (ALL A and ALL B) and one that had not (ALL C). Fig. 4 shows the results of these studies. In general for ALL A and ALL B the combination of plerixafor and chemotherapy drug produced greater antileukemia effect than plerixafor or chemotherapy drug alone. We did not see similar enhanced toxicity with ALL C.

We analyzed the data for ALL A and B (in which we consistently saw enhanced chemotherapy drug activity in the presence of plerixafor) to determine if the effects were additive or synergistic. For mercaptopurine and for methotrexate the observed effect of combined treatment with plerixafor was greater than the fractional product prediction which suggested that synergistic interactions between the drugs were occurring (data not shown).

Recombinant CXCL12 cannot substitute for stromal cell support of leukemia cells. The modest effects we saw with CXCL12 blockade suggested that the mechanisms of stromal cell support were multifactorial. To test this we performed three experiments with three different leukemias in which recombinant CXCL12 was present in the culture medium. For two of the leukemias CXCL12 in the presence of stroma increased the number of surviving leukemia cells. However, in the absence of stroma CXCL12 did not produce any improvement of leukemia cell survival (Fig. 5). 

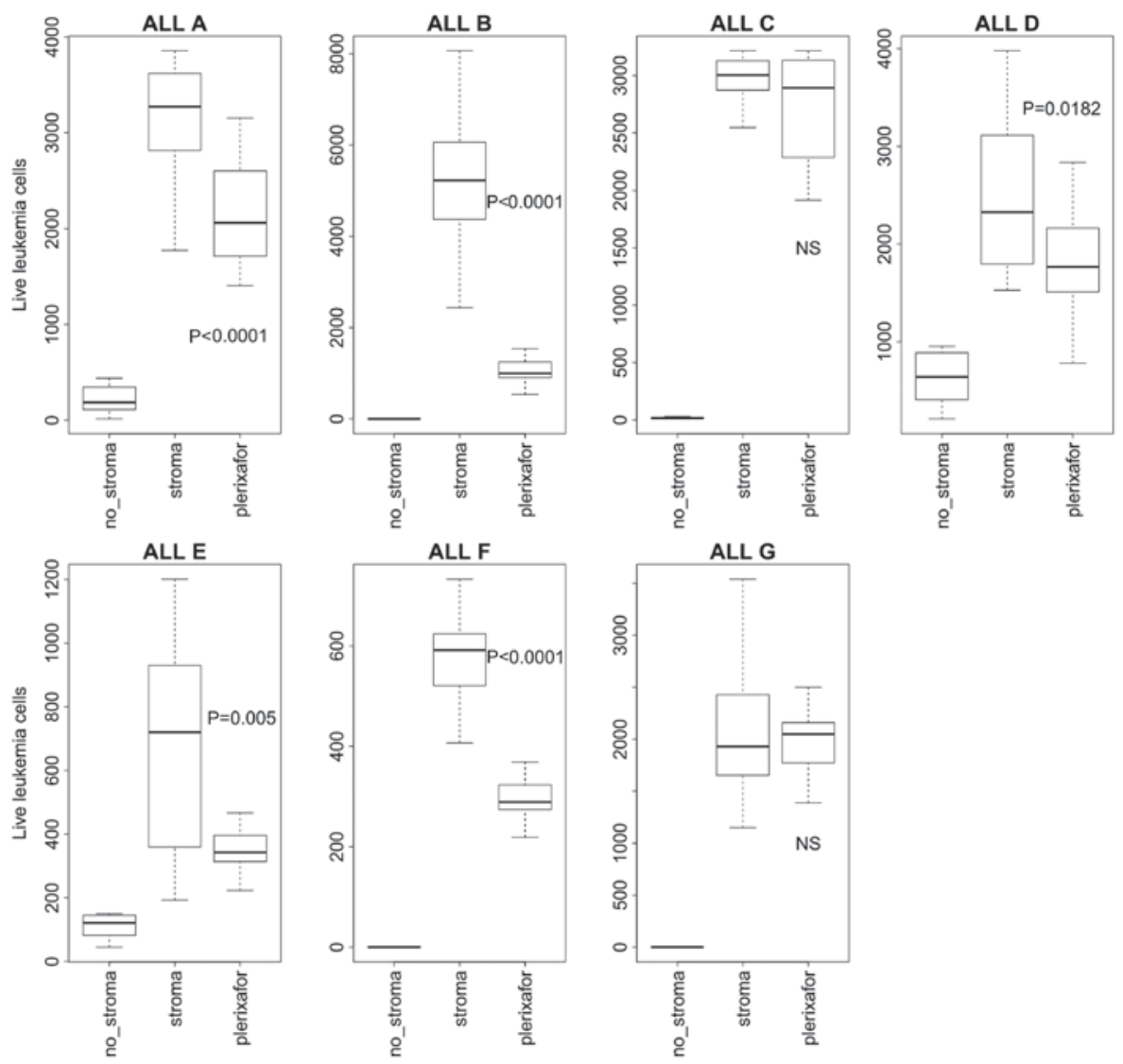

Figure 2. Effect of plerixafor on survival of ALL cells supported by stromal cells. Each panel represents the number of leukemia cells surviving in wells with no stroma ( $\mathrm{n}=12-18)$, stroma ( $\mathrm{n}=12-17)$, or stroma with plerixafor $200 \mu \mathrm{M}(\mathrm{n}=12-18)$. Each panel represents a unique ALL. Box and whisker plots represent the data. P-values are for comparisons of stroma with and without plerixafor. ALL, acute lymphoblastic leukemia; NS, no significant difference.

Gene set enrichment analysis. The modest impact of interfering with CXCL12 is consistent with the hypothesis that the mechanisms of stromal support for leukemia involve many genes that contribute to the overall effect. To gain some insight into this we performed gene set enrichment analysis. In the first analysis we compared gene expression profiles between cells that efficiently supported leukemia cell survival and those cells that did not support leukemia cells survival (see Table II for the list of the cells in these groups). Within the set of genes overrepresented among cells that support leukemia we observed several related molecular functions (Table III). Not surprisingly we observed a number of functions related to extracellular matrix. More interestingly we saw enrichment of growth factor related genes including platelet-derived growth factor and insulin-like growth factor. We also unexpectedly observed enrichment of molecular functions related to redox and energy metabolism.

We performed a second analysis in which we compared gene expression profiles before and after ALL A leukemia cells physically interacted with stromal cells. After contact with leukemia cells we observed 373 differentially expressed genes in stromal cells. Genes related to redox reactions and energy metabolism were prominently overrepresented, as were genes related to growth factor and glycosaminoglycan binding (Table III). After contact with stromal cells we observed 643 genes differentially expressed in the ALL A leukemia cells. Again we saw enrichment of molecular functions related to redox, to interactions with the extracellular matrix, and to growth factor binding. We also saw overrepresentation of genes related to TRAIL binding (Table III).

Leukemia cells and stroma cells exchange intracellular materials. The unexpected prominence of genes related to redox reactions and energy metabolism in both stromal and leukemia cells, coupled with our earlier observation that direct cell-cell contact was essential for stromal support of leukemia cells, suggested the possibility that the cells may be exchanging intracellular materials related to or affecting metabolism. To test this hypothesis we labeled cells with calcein AM which is a cell permeant dye of molecular weight $<1000$ Daltons that in living cells is converted to a fluorescent moiety. In these studies we used the HS27 immortalized stromal cell line or primary bone marrow stromal cells. We examined five of the leukemias (ALL A, ALL B, ALL C, ALL F and ALL G). We then incubated either unlabeled leukemia cells with labeled stromal cells, or unlabeled stromal cells with labeled leukemia cells. $24 \mathrm{~h}$ later we performed flow cytometry to detect intracellular fluorescent calcein. Representative results are presented in Fig. 6. We observed transfer of calcein from leukemia cells to stromal cells (Fig. 6D), and also observed transfer of label from stromal cells to leukemia cells (Fig. 6B). We repeated these experiments using Transwell barriers in which leukemia cells and stromal cells were in the same well but were unable to establish cell:cell contact. When stromal cells and leukemia cells could were not in physical contact we did not see transfer of calcein (Fig. 6A and 6C). 
A CXCR4

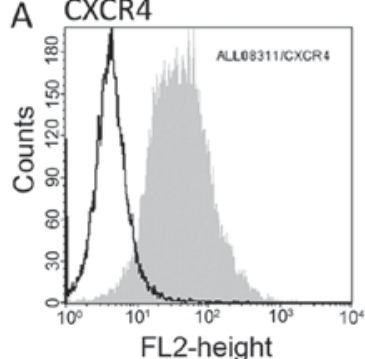

ALL A

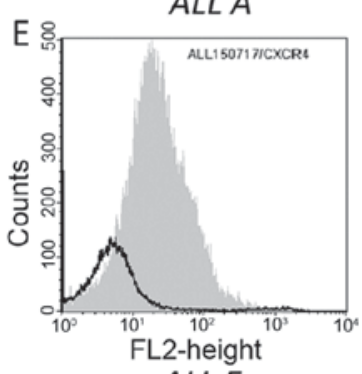

ALL E
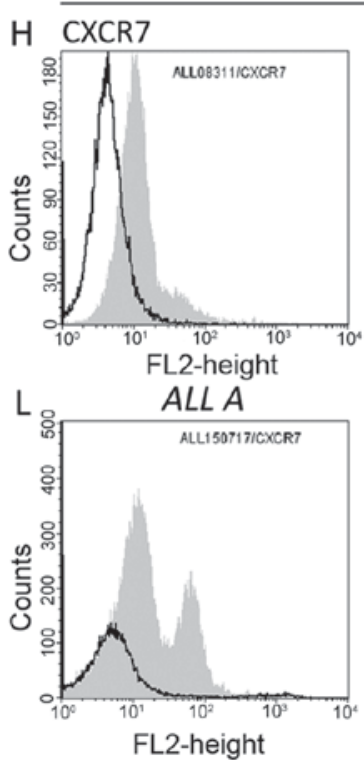

ALLE
B
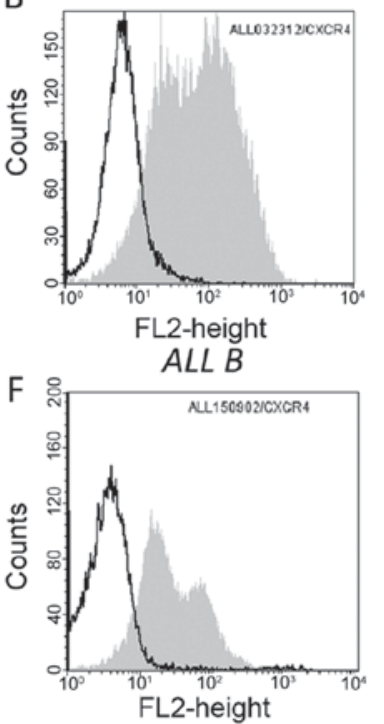

ALL F
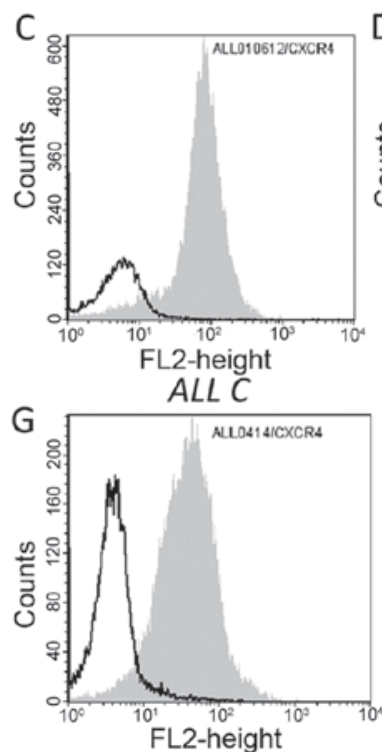

ALL G

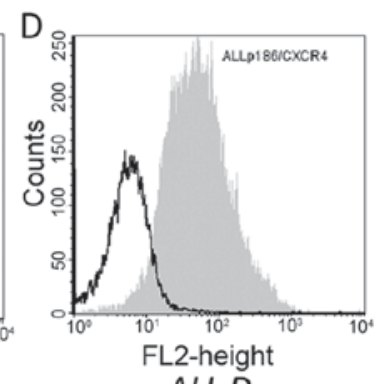

ALL D
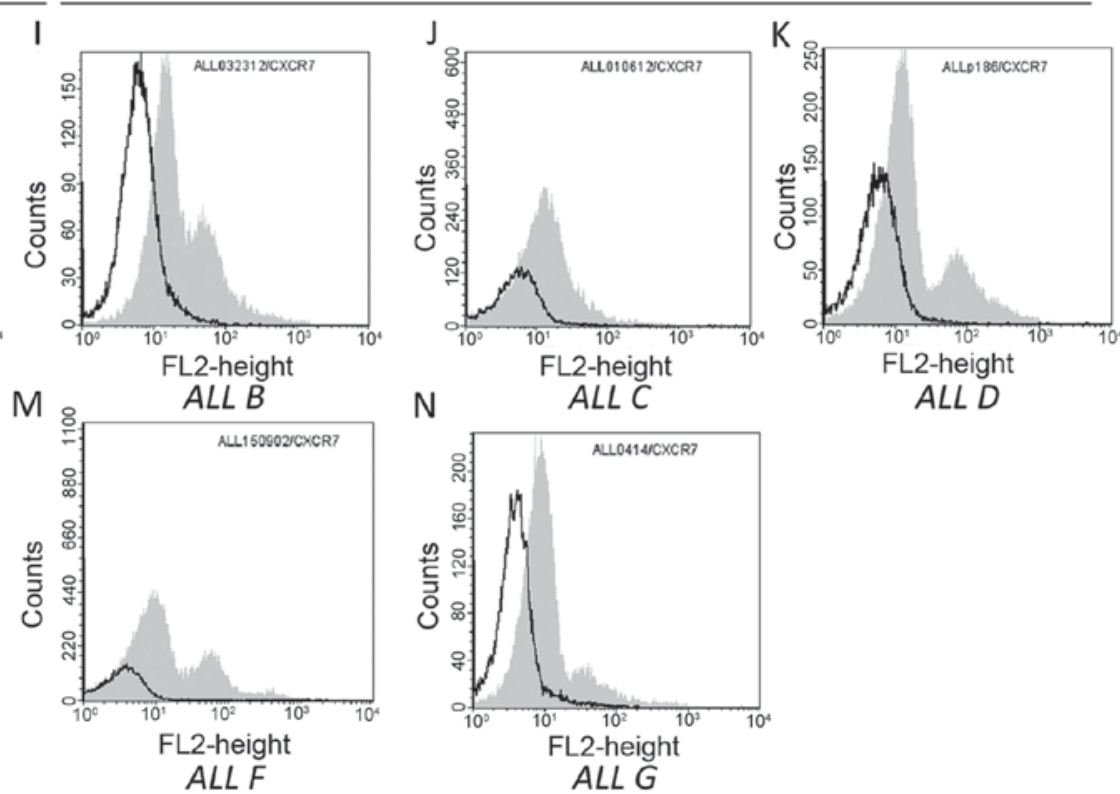

ALL D

Figure 3. CXCR4 and CXCR7 expression in ALL cells. Flow cytometry for (A-G) CXCR4 and (H-N) CXCR7. Letter in each histogram identifies the ALL. Forward and side scatter gates were used to identify intact leukemia cells. Shaded histograms are specific antibody while unfilled histograms are isotype control. CXCR, C-X-C chemokine receptor type; ALL, acute lymphoblastic leukemia.

Leukemia cells and stroma cell exchange mitochondria. Mitochondria play critical roles in cellular energy metabolism. Since we had observed that genes related to energy metabolism and redox reactions were associated with stromal cell support of leukemia, and that cell-cell contact was critical for leukemia cell survival, we hypothesized that mitochondria might play a role. To assess this we treated either stromal cells or leukemia cells (ALL B or ALL F) with the vital dye MitoTracker Red which accumulates in active mitochondria. Labeled cells were added to unlabeled cells, and then examined by fluorescence microscopy the next day. We observed transfer of mitochondria from labeled leukemia cells to unlabeled stromal cell; we also observed transfer of mitochondria from labeled stromal cells to unlabeled leukemia cells (Fig. 7). Separation of cells by a Transwell prevented mitochondrial transfer (data not shown).

\section{Discussion}

Most cancers are mutationally diverse and the effort to develop leukemia pathway-specific or leukemia mutation-specific may lead to potent drugs that are active against only a fraction of leukemias. Mutationally diverse leukemias appear to share a common dependence on nonmalignant stroma. In principle, agents directed against common mechanisms of microenvironmental support might exhibit activity against mutationally diverse leukemias. Stromal cell-derived CXCL12 has been identified as one potential mechanism. Our study critically assessed the potential utility of targeting CXCL12 in a panel of recently derived patient-derived ALL xenografts in vitro. Our observations confirm observations by others that CXCL12 may play a role in keeping ALL cells alive $(17,18)$. However, our findings extend these observations 

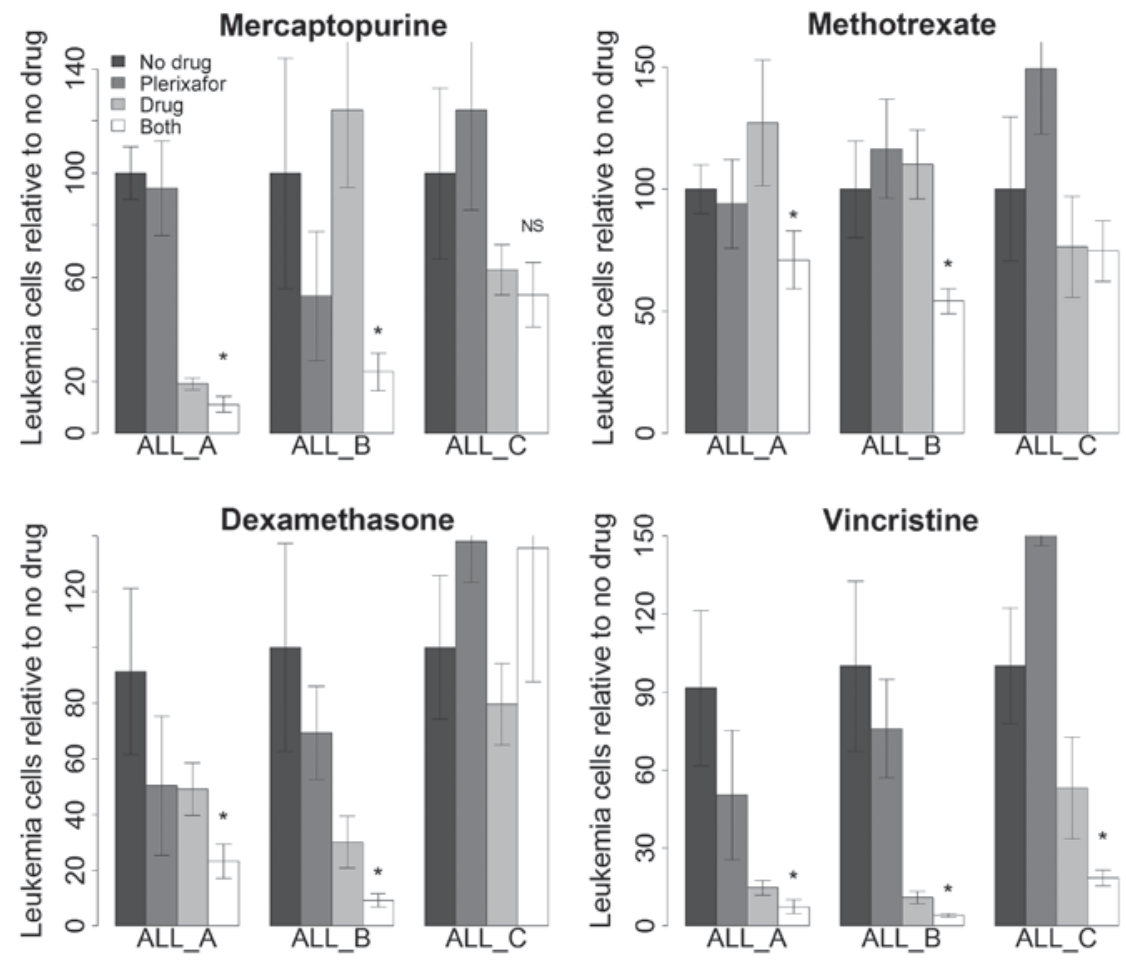

Figure 4. Effect of plerixafor on antileukemia activity of conventional chemotherapy drugs. Each panel represents a different chemotherapy drug with or without plerixafor. For each drug several ALLs were studied (ALLs A, B and C). Box and whisker plots represent the data. Bars represent live the average number of live ALL cells normalized to 'no drug'. Error bars represent 95 percentile confidence intervals of the mean. From left to right the bars represent no drug (black, n=8-12), plerixafor alone (dark gray, $n=8-12$ ), chemotherapy drug alone (light gray, $n=8-12$ ), or the combination of both (white, $n=8-12$ ). $T$ tests were performed comparing 'drug alone' to 'both drug and plerixafor'. "P $<0.05$ vs. drug alone. NS, significant; ALL, acute lymphoblastic leukemia.
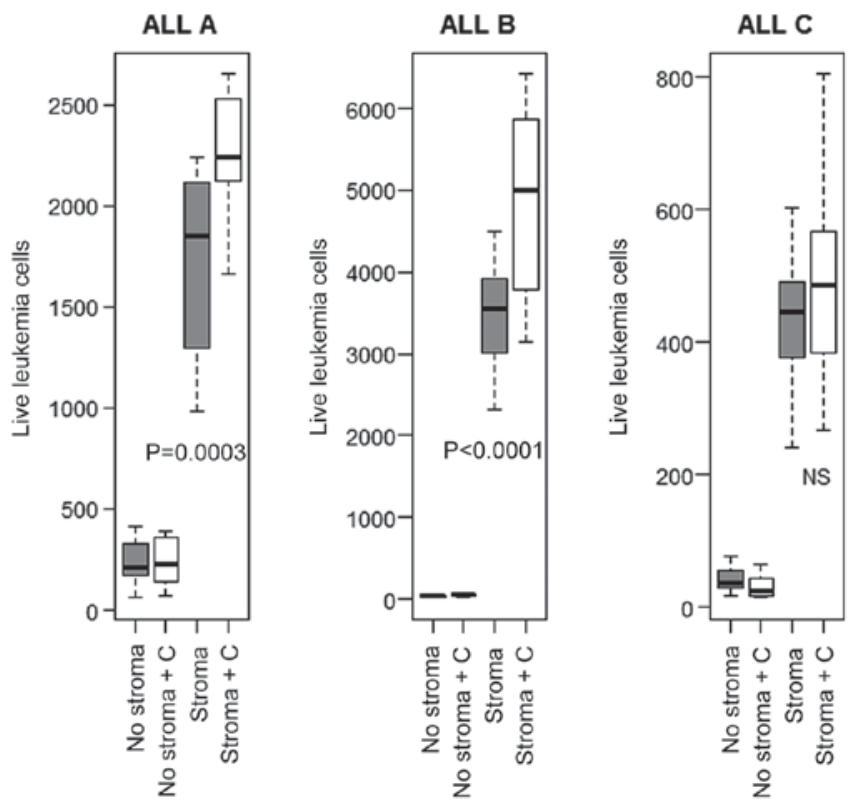

Figure 5. CXCL12 in the absence of stromal cells does not prevent leukemia cell death. Recombinant CXCL12 was added to microwells with ALL cells in the presence $($ stroma $+\mathrm{C}$ ) or absence (no stroma $+\mathrm{C}$ ) of stroma. Controls included wells without stroma or CXCL12 ('no stroma') or stroma without CXCL12 (stroma). Three unique ALL's were used. A total of 13-18 replicates per condition were used. Box and whiskers plots are presented. P-values are from t tests. NS, not significant; ALL, acute lymphoblastic leukemia; CXCL12, C-X-C motif chemokine ligand 12.

by showing that the effect was not universal in ALL as 2 of the 7 leukemias were unaffected by plerixafor. Moreover, our observations show that heterogeneity of the effect was not closely associated with leukemia cell surface expression of either CXCR4 or CXCR7, another receptor for CXCL12 $(15,16)$.

We assessed the potential interaction of CXCL12 blockade by plerixafor with drugs commonly used in the treatment of ALL. When used with leukemias more sensitive to plerixafor alone (e.g., ALL A and ALL B) we saw synergistic antileukemia effects for most conventional drugs with plerixafor. One should note that the conventional drugs (mercaptopurine, methotrexate, dexamethasone and vincristine) have different antileukemia mechanisms, but all ultimately trigger apoptosis in damaged cells. This suggests the hypothesis that the plerixafor interference with CXCL12 mediated stromal support of leukemia may lower the apoptotic threshold for drug-damaged cells. Our results confirm the observations of several other groups that CXCR4 blockade can enhance the antileukemia activity of conventional chemotherapy (17,19-23). Our results extend these observations by demonstrating that the effect of plerixafor may be a synergistic effect with conventional chemotherapy. A recent phase I trial of plerixafor in combination with cytarabine and etoposide reported biological responses but modest clinical responses in a heavily pretreated group of patients with ALL, acute myelogenous leukemia or myelodysplastic syndrome (24).

The mechanisms of CXCL12 on hematopoietic or leukemia cell are not fully elucidated. CXCL12 (also known as SDF1, stromal cell-derived factor 1) has been extensively investigated and is related to many cellular functions related to development, immune responses and tumor growth and metastasis. In hematology it is best known as a factor that contributes to 
Table III. Gene set enrichment analysis.

A, Analysis of genes differentially expressed in stromal cells compared to cells that do not support ALL

Stromal cells that support

leukemia gene enrichment
Percentage of

genes involved
Multiple comparison adjusted p-value

Cellular components

Extracellular matrix

Cell surface

14

11

27

Molecular function

Growth factor binding

PDGF binding

Receptor binding

Biological process

Extracellular matrix organization

Biological adhesion

Regulation of cell death

$\begin{array}{ll}14 & 5.10 \times 10^{-25} \\ 11 & 2.60 \times 10^{-09} \\ 27 & 1.30 \times 10^{-10} \\ 6 & \\ 2 & 5.20 \times 10^{-12} \\ 15 & 1.60 \times 10^{-06} \\ & 2.60 \times 10^{-06} \\ 14 & \\ 18 & 7.70 \times 10^{-25} \\ 14 & 5.30 \times 10^{-17} \\ & 6.10 \times 10^{-04}\end{array}$

B, Analysis of genes differentially expressed in ALL cells after contact with stromal cells

ALL leukemia cell genes changed by contact with stroma gene enrichment
Percentage of genes involved
Multiple comparison adjusted p-value

Cellular components

Extracellular region

Extracellular vesicle

35

28

27

Extracellular membrane bound organelle

Molecular function

Protein binding

Purine nucleotide binding

Small molecule binding

Biological process

Negative regulation of biological process

Negative regulation of apoptotic process

Cellular response to cytokine stimulus
$1.60 \times 10^{-12}$

$3.70 \times 10^{-16}$

$3.70 \times 10^{-16}$

$2.30 \times 10^{-11}$

$3.10 \times 10^{-05}$

$9.20 \times 10^{-06}$

$1.40 \times 10^{-14}$

$3.30 \times 10^{-05}$

$3.40 \times 10^{-05}$

C, Analysis of genes differentially expressed in stroma cells after contact with ALL cells

Stromal genes changed by contact with ALL leukemia gene enrichment
Percentage of genes involved
Multiple comparison adjusted p-value

Cellular components

Extracellular region

Extracellular vesicle

Molecular function

Oxidoreductases

NAD/NADP/alcohol metabolism

Daunorubicin metabolism

Biological process

Interferon signaling

Regulation of cell proliferation

Primary alcohol metabolic process
$3.30 \times 10^{-09}$

$1.30 \times 10^{-06}$

$1.30 \times 10^{-06}$

$1.30 \times 10^{-04}$

$1.30 \times 10^{-04}$

$1.30 \times 10^{-02}$

$5.70 \times 10^{-19}$

$2.70 \times 10^{-07}$

$5.20 \times 10^{-08}$

ALL, acute lymphoblastic leukemia. 


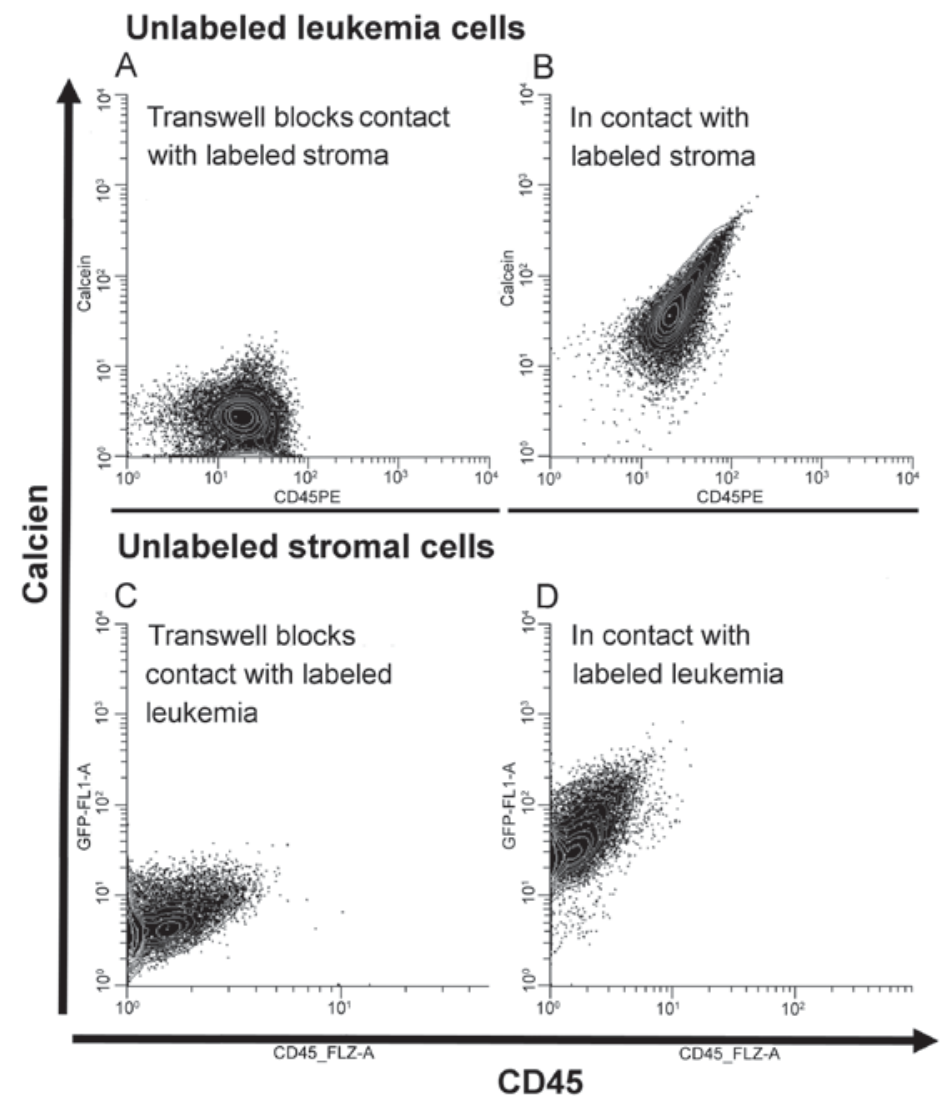

Figure 6. Intercellular transfer of calcien between stromal cells and leukemia cells requires cell-cell contact. Flow cytometry was performed after $24 \mathrm{~h}$ of coculture of stromal cells and leukemia cells. (A and B) Primary bone marrow stromal cells were labeled with calcien. Unlabeled ALL F leukemia cells were added to stromal cultures (A) with a Transwell or (B) without a Transwell. (A and B) Live leukemia cells were identified by scatter characteristics and exclusion of AAD. (C and D) ALL A leukemia cell were labeled while HS27 stromal cells were unlabeled. (C) Transwell separated cells. (D) No Transwell was used and leukemia and stromal cells were in direct contact. (C and D) Live stromal cells were identified by scatter characteristics and exclusion of AAD. ALL, acute lymphoblastic leukemia; AAD, actinomycin D; CD45, cluster of differentiation 45.

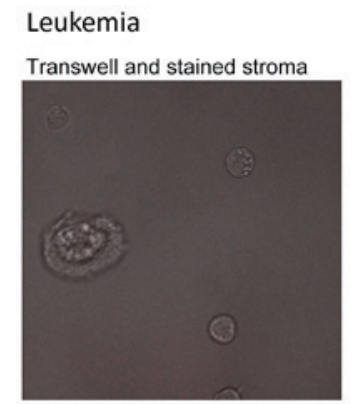

Stroma

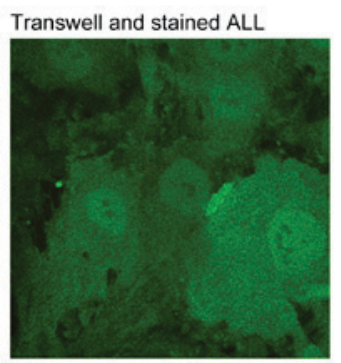

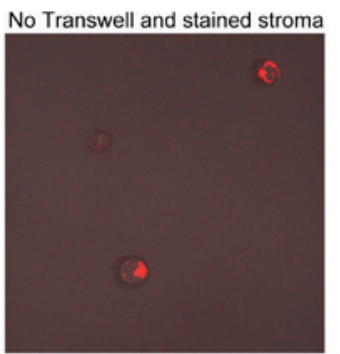

No Transwell and stained ALL

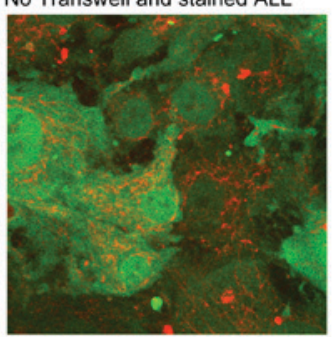

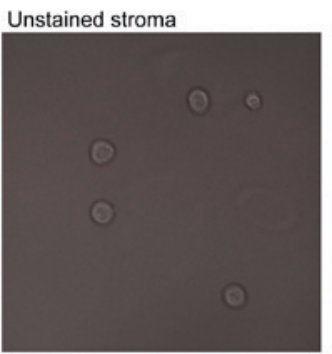

Unstained ALL

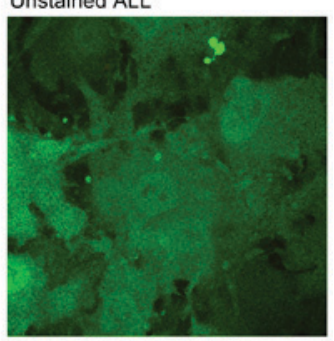

Figure 7. Transfer of labeled mitochondria between leukemia and stromal cells after 24 h of coculture. Fluorescence confocal microscopy (magnification, x100) was used to examine mitochondria stained with MitoTracker Red CMXRos. The leukemia cells were ALL F, and the stromal cells were the hTERT immortalized GFP+ stromal cells. Top panels: Leukemia cells imaged by merged brightfield and red fields. Top left: Leukemia cells which had been in wells for $24 \mathrm{~h}$ with stained stromal cells but direct cell contact was prevented by Transwell. Top middle: Leukemia cells after direct $24-\mathrm{h}$ contact with stained stroma. Top right: Leukemia cells after 24-hour contact with unstained stroma. Bottom panels: Green fluorescent protein-expressing stromal cells imaged by merged green and red fields. Bottom left: Stromal cells after coculture with stained leukemia cells by separated by Transwells. Bottom middle: Stromal cells after direct contact with stained leukemia cells. Bottom right: Stromal cells after direct contact with unstained leukemia cells. ALL, acute lymphoblastic leukemia; hTERT, human telomoerase gene. 
angiogenesis and regulates hematopoiesis and hematopoietic progenitor cell homing in the marrow (25-27). One theme of these reports on the mechanism is that CXL12 plays a role in the hematopoietic cell homing and efficient binding of hematopoietic cells to stroma through adhesion molecules (6-9).

One model of stromal cell-leukemia cell microenvironmental interaction is that stromal cells present to leukemia cells ligands or growth factors (such as CXCL12) on the cell surface or in the intracellular region. The modest effect we saw with plerixafor plus the biological themes that emerged from the gene expression analyses led us to step back from this model. To our surprise we observed changes in genes related to energy metabolism and redox status, processes that are intracellular. This led us to broaden the hypothesis that stromal cells provided metabolic support to leukemia cells and that this metabolic support contributed to leukemia cell survival. Central to this hypothesis is the need to demonstrate some means by which stromal cells and leukemia cells can exchange metabolites. Our experiments in which we observed bidirectional transfer of calcien between stromal cells and leukemia cells established that this is possible.

Given the prominence of pathways related to energy metabolism and redox reactions in the gene set enrichment analyses we also hypothesized that intercellular mitochondria exchange may be involved. Intercellular transport of organelles between cells though tunneling nanotubes was described $>10$ years ago (28). Exchange of functioning mitochondria and improved aerobic respiration in recipient cells has also been observed (29). Exchange of mitochondria between malignant cells and nonmalignant cells has also been observed (30-34). Our observation of bidirectional transfer between nonmalignant stromal cells and ALL cells is consistent with these observations in other systems.

There are limitations in this approach to dissecting the mechanisms by which nonmalignant stromal cells provide support to ALL cells. The first is the simplicity of our coculture system. In the living marrow environment there are many types of cells interacting including a variety of cells derived from mesenchymal stem cells, cells derived from normal hematopoietic stem cells and leukemia cells. Our in vitro model is very simple and does not fully recreate the in vivo microenvironment. The second is that the stromal cells and leukemia cells are from different humans. It is possible there are polymorphisms in a number of functions that may obscure critical interdependencies. The third is the lack of knowledge of the genetic abnormalities in the ALLs studied. If one wished to apply this or a similar system to define novel therapeutic targets, such knowledge would be very helpful in defining which ALL's might be successfully targeted.

\section{Acknowledgements}

Not applicable.

\section{Funding}

The present study was supported by grants from the St. Baldrick's Foundation (Monrovia, CA, USA; to CAM) and Alex's Lemonade Stand Foundation for Childhood Cancer (Wynnewood, PA, USA; to CAM).

\section{Availability of data and materials}

The datasets used and/or analyzed during the current study are available from the corresponding author on reasonable request.

\section{Authors' contributions}

SU designed and performed experiments, interpreted data and wrote an initial draft of the manuscript. US, OT and LN designed, performed and interpreted experiments. JCS assisted with data interpretation and manuscript editing. CAM was responsible for the overall project, designed experiments, interpreted data and wrote the final version of the manuscript. All authors read and approved the final manuscript.

\section{Ethics approval and consent to participate}

The Research Subjects Review Board of the University of Rochester approved the use of the human tissues in the present study (RSRB00046358, approval dates 2/28/2013 to present). The University Committee on Animal Resources approved the use of mice used in these studies (UCAR 102081/UCAR-2003-237E, approval dates 2003 to present).

\section{Patient consent for publication}

Not applicable.

\section{Competing interests}

The authors declare that they have no competing interests.

\section{References}

1. Shahrabi S, Rezaeeyan H, Ahmadzadeh A, Shahjahani M and Saki N: Bone marrow blood vessels: Normal and neoplastic niche. Oncol Rev 10: 306, 2016.

2. Ramasamy SK: Structure and Functions of Blood Vessels and Vascular Niches in Bone. Stem Cells Int 2017: 5046953, 2017.

3. Manabe A, Coustan-Smith E,Behm FG, Raimondi SC and Campana D: Bone marrow-derived stromal cells prevent apoptotic cell death in B-lineage acute lymphoblastic leukemia. Blood 79: 2370-2377, 1992.

4. Manabe A, Murti KG, Coustan-Smith E, Kumagai M, Behm FG, Raimondi SC and Campana D: Adhesion-dependent survival of normal and leukemic human B lymphoblasts on bone marrow stromal cells. Blood 83: 758-766, 1994.

5. Dominici M, Le Blanc K, Mueller I, Slaper-Cortenbach I, Marini F, Krause D, Deans R, Keating A, Prockop DJ and Horwitz E: Minimal criteria for defining multipotent mesenchymal stromal cells. The International Society for Cellular Therapy position statement. Cytotherapy 8: 315-317, 2006.

6. Glodek AM, Le Y, Dykxhoorn DM, Park SY, Mostoslavsky G, Mulligan R, Lieberman J, Beggs HE, Honczarenko M and Silberstein LE: Focal adhesion kinase is required for CXCL12-induced chemotactic and pro-adhesive responses in hematopoietic precursor cells. Leukemia 21: 1723-1732, 2007.

7. Acharya M, Edkins AL, Ozanne BW and Cushley W: SDF-1 and PDGF enhance alphavbeta5-mediated ERK activation and adhesion-independent growth of human pre-B cell lines. Leukemia 23: 1807-1817, 2009.

8. Arnaud MP, Vallée A, Robert G, Bonneau J,Leroy C, Varin-Blank N, Rio AG, Troadec MB, Galibert MD and Gandemer V: CD9, a key actor in the dissemination of lymphoblastic leukemia, modulating CXCR4-mediated migration via RAC1 signaling. Blood 126: 1802-1812, 2015.

9. Shen N, Ffrench P, Guyotat D, Ffrench M, Fiere D, Bryon PA and Dechavanne M: Expression of adhesion molecules in endothelial cells during allogeneic bone marrow transplantation. Eur J Haematol 52: 296-301, 1994. 
10. Mihara K, Imai C, Coustan-Smith E, Dome JS, Dominici M Vanin E and Campana D: Development and functional characterization of human bone marrow mesenchymal cells immortalized by enforced expression of telomerase. $\mathrm{Br} \mathrm{J}$ Haematol 120: 846-849, 2003.

11. Roecklein BA and Torok-Storb B: Functionally distinct human marrow stromal cell lines immortalized by transduction with the human papilloma virus E6/E7 genes. Blood 85: 997-1005, 1995.

12. Greco WR, Bravo G and Parsons JC: The search for synergy: A critical review from a response surface perspective. Pharmacol Rev 47: 331-385, 1995.

13. To LB, Levesque JP and Herbert KE: How I treat patients who mobilize hematopoietic stem cells poorly. Blood 118: 4530-4540, 2011.

14. Fricker SP: Physiology and pharmacology of plerixafor. Transfus Med Hemother 40: 237-245, 2013.

15. Sánchez-Martín L, Sánchez-Mateos $P$ and Cabañas C: CXCR7 impact on CXCL12 biology and disease. Trends Mol Med 19: 12-22, 2013.

16. Asri A, Sabour J, Atashi A and Soleimani M: Homing in hematopoietic stem cells: Focus on regulatory role of CXCR7 on SDF1a/CXCR4 axis. EXCLI J 15: 134-143, 2016.

17. Sison EA, Rau RE, McIntyre E, Li L, Small D and Brown P: MLL-rearranged acute lymphoblastic leukaemia stem cell interactions with bone marrow stroma promote survival and therapeutic resistance that can be overcome with CXCR4 antagonism. Br J Haematol 160: 785-797, 2013.

18. Nishii K, Katayama N, Miwa H, Shikami M, Masuya M, Shiku H and Kita K: Survival of human leukaemic B-cell precursors is supported by stromal cells and cytokines: Association with the expression of bcl-2 protein. Br J Haematol 105: 701-710, 1999.

19. Juarez J, Bradstock KF, Gottlieb DJ and Bendall LJ: Effects of inhibitors of the chemokine receptor CXCR4 on acute lymphoblastic leukemia cells in vitro. Leukemia 17: 1294-1300, 2003.

20. Welschinger R, Liedtke F, Basnett J, Dela Pena A, Juarez JG, Bradstock KF and Bendall LJ: Plerixafor (AMD3100) induces prolonged mobilization of acute lymphoblastic leukemia cells and increases the proportion of cycling cells in the blood in mice. Exp Hematol 41: 293-302 e291, 2013.

21. Sison EA, Magoon D, Li L, Annesley CE, Rau RE, Small D and Brown P: Plerixafor as a chemosensitizing agent in pediatric acute lymphoblastic leukemia: Efficacy and potential mechanisms of resistance to CXCR4 inhibition. Oncotarget 5: 8947-8958, 2014

22. Sison EA, Magoon D, Li L, Annesley CE, Romagnoli B, Douglas GJ, Tuffin G, Zimmermann J and Brown P: POL5551, a novel and potent CXCR4 antagonist, enhances sensitivity to chemotherapy in pediatric ALL. Oncotarget 6: 30902-30918, 2015.

23. Randhawa S, Cho BS, Ghosh D, Sivina M, Koehrer S, Müschen M, Peled A, Davis RE, Konopleva M and Burger JA: Effects of pharmacological and genetic disruption of CXCR4 chemokine receptor function in B-cell acute lymphoblastic leukaemia. Br J Haematol 174: 425-436, 2016.
24. Cooper TM, Sison EAR, Baker SD, Li L, Ahmed A, Trippett T, Gore L, Macy ME, Narendran A, August K, et al: A phase 1 study of the CXCR4 antagonist plerixafor in combination with high-dose cytarabine and etoposide in children with relapsed or refractory acute leukemias or myelodysplastic syndrome: A Pediatric Oncology Experimental Therapeutics Investigators Consortium study (POE 10-03). Pediatr Blood Cancer: doi.10.1002/pbc.26414.

25. Anthony BA and Link DC: Regulation of hematopoietic stem cells by bone marrow stromal cells. Trends Immunol 35: 32-37, 2014.

26. Karpova D and Bonig H: Concise Review: CXCR4/CXCL12 signaling in immature hematopoiesis-lessons from pharmacological and genetic models. Stem Cells 33: 2391-2399, 2015.

27. Gomes AC and Gomes MS: Hematopoietic niches, erythropoiesis and anemia of chronic infection. Exp Hematol 44: 85-91, 2016.

28. Rustom A, Saffrich R, Markovic I, Walther P and Gerdes HH: Nanotubular highways for intercellular organelle transport. Science 303: 1007-1010, 2004.

29. Spees JL, Olson SD, Whitney MJ and Prockop DJ: Mitochondrial transfer between cells can rescue aerobic respiration. Proc Natl Acad Sci USA 103: 1283-1288, 2006.

30. Wang $\mathrm{X}$ and Gerdes $\mathrm{HH}$ : Transfer of mitochondria via tunneling nanotubes rescues apoptotic PC12 cells. Cell Death Differ 22: 1181-1191, 2015.

31. Lou E, Fujisawa S, Morozov A, Barlas A, Romin Y, Dogan Y, Gholami S, Moreira AL, Manova-Todorova K and Moore MA: Tunneling nanotubes provide a unique conduit for intercellular transfer of cellular contents in human malignant pleural mesothelioma. PLoS One 7: e33093, 2012.

32. Pasquier J, Guerrouahen BS, Al Thawadi H, Ghiabi P, Maleki M, Abu-Kaoud N, Jacob A, Mirshahi M, Galas L, Rafii S, et al: Preferential transfer of mitochondria from endothelial to cancer cells through tunneling nanotubes modulates chemoresistance. J Transl Med 11: 94, 2013.

33. Moschoi R, Imbert V, Nebout M, Chiche J, Mary D, Prebet T, Saland E, Castellano R, Pouyet L, Collette Y, et al: Protective mitochondrial transfer from bone marrow stromal cells to acute myeloid leukemic cells during chemotherapy. Blood 128: 253-264, 2016

34. Marlein CR, Zaitseva L, Piddock RE, Robinson SD, Edwards DR, Shafat MS, Zhou Z, Lawes M, Bowles KM and Rushworth SA: NADPH oxidase-2 derived superoxide drives mitochondrial transfer from bone marrow stromal cells to leukemic blasts. Blood 130: 1649-1660, 2017.

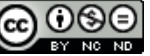

This work is licensed under a Creative Commons

Attribution-NonCommercial-NoDerivatives

4.0

International (CC BY-NC-ND 4.0) License. 